\title{
Performance of the DDD as a Direct Electron Detector for Low Dose Electron Microscopy
}

\author{
L. Jin,* and R. Bilhorn,* \\ * Direct Electron, LP, 13240 Evening Creek Drive S., Suite 311, San Diego, CA 92128
}

In recent years, low dose electron microscopy techniques, including single particle CryoEM and Cryo-Electron Tomography [1,2], have become powerful tools for studying macromolecular assemblies. These techniques require extremely low total electron dose, which proves to be a challenging imaging condition for CCD based camera systems that rely on phosphor scintillators coated on fused fiber optic bundles that are bonded to the CCD.

A new type of detector, that is capable of direct electron detection, holds great promise for dramatically improving the image quality for low dose EM images [3-6]. In this report, we introduce the principles of the Direct Detection Device $\left(\mathrm{DDD}^{\circledR}\right)$, describe the performance of the 12 megapixel DDD camera (DE-12), and compare the results with film as well as with scintillator-based CCD systems.

FIG. 1 shows Detective Quantum Efficiency (DQE) curves for the DE-12 camera system measured at 300 and $400 \mathrm{keV}$. DQE is an objective method for comparing performance of different camera systems. In this case a precision edge is used to create a shadow image from which the measured Edge Spread Function (ESF) is used to calculate the Modulation Transfer Function (MTF). The $\mathrm{DQE}$ at zero spatial frequency was calculated using the noise binning method and the microscope beam intensity was measured using a Faraday plate with applied backscattering correction. The details of the calculation can be found in references [7,8]. DQE values 3 to 4 times higher than those for $\mathrm{CCD}$ based systems at frequencies at and beyond the half-Nyquist frequency are the basis for the tremendous performance advantage of the $\mathrm{DDD}^{\circledR}$.

FIG. 2 shows three images of a grid with GroEL and TMV in ice taken with the DE-12 camera using the automated Multi-Scale Imaging (MSI) feature in the Leginon software package [9]. The data was acquired on a FEI Tecnai Spirit $120 \mathrm{kV}$ microscope at the National Resource for Automated Molecular Microscopy at the Scripps Research Institute. The high resolution image taken at 21000x magnification (2.3 Angstrom/pixel) was cropped to show the TMV. Total electron dose in the final image is $\sim 11 \mathrm{e}^{-} /$Angstrom $^{2}$. As these images demonstrate, the high SNR of the DE-12 camera provides excellent contrast from inherently low-contrast specimens while the high sensitivity and large array size allow coverage of large specimen areas at low dose.

\section{References}

[1] J. Frank, Annu. Rev. Biophys. Biomol. Struct. 31 (2002) 303-319.

[2] S. Subramaniam and J.L.S. Milne, Annu. Rev. Biophys. Biomol. Struct. 33 (2004) 141-155.

[3] A.C. Milazzo, et al., Ultramicroscopy 104 (2005) 152-159.

[4] L. Jin, et al., Journal of structural biology 161 (2008) 352-358.

[5] G. McMullan and A.R. Faruqi, Nucl. Instrum. Meth. A 591 (2008) 129-133. 
[6] G. Deptuch, et al., Ultramicroscopy 107 (2007) 674-684.

[7] R.R. Meyer and A.I. Kirkland, Microsc. Res. Tech. 49 (2000) 269.

[8] G. McMullan, et al., Ultramicroscopy 109 (2009) 1126-1143.

[9] C. Suloway, et al., Journal of structural biology 151 (2005) 41-60.

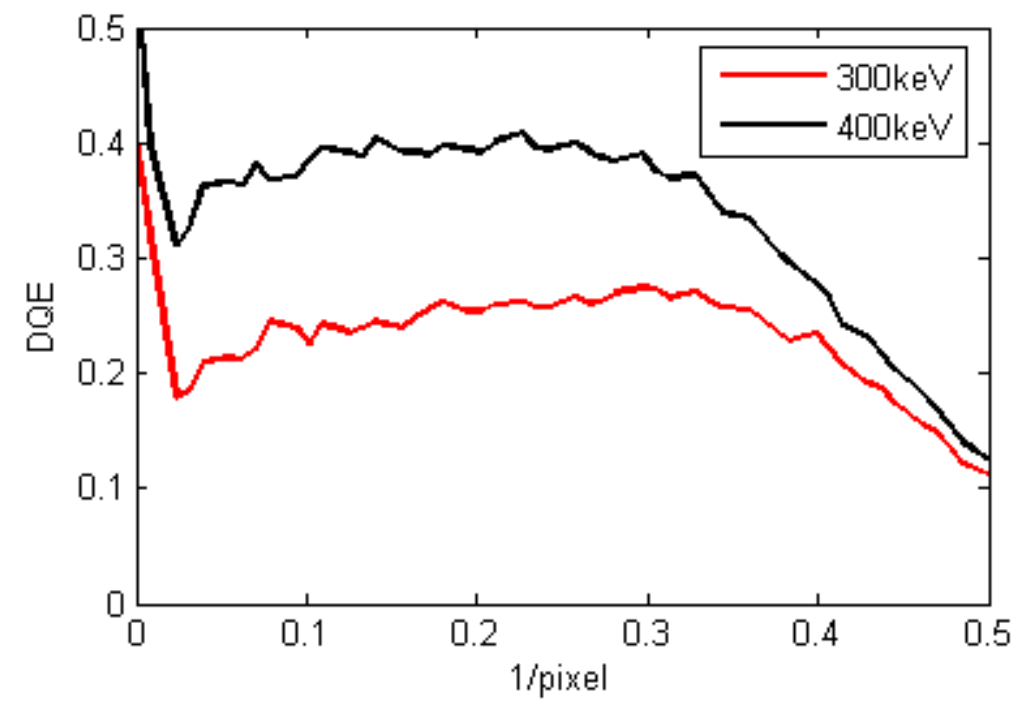

FIG. 1. DQE for the DE-12 DDD ${ }^{\circledR}$ camera system at electron acceleration energies of $300 \mathrm{kV}$ and 400kV. JEOL 4000EX, National Center for Microscopy and Imaging Research at the University of California, San Diego (UCSD).

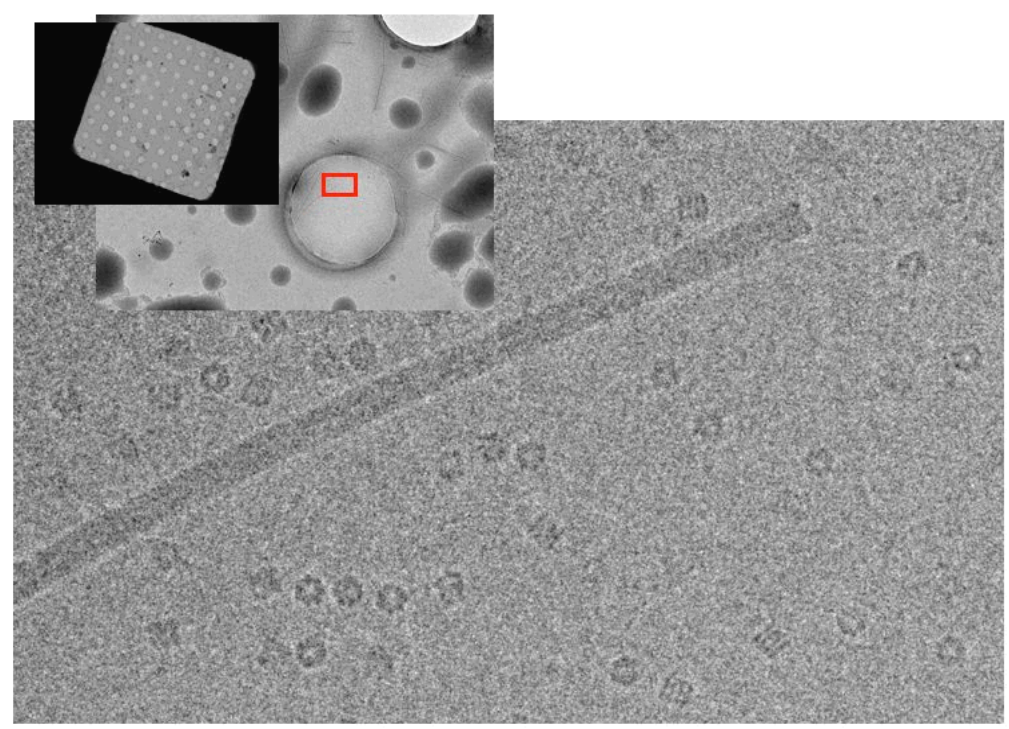

FIG. 2. Automated Multi-Scale Imaging of GroEL and TMV acquired at $120 \mathrm{kV}$ using Leginon. Images were recorded at 290x, 2700x, and 21000x magnifications on a FEI Tecnai Spirit at the National Resource for Automated Molecular Microscopy at the Scripps Research Institute. 